\title{
Parasite infection impairs the shoaling behaviour of uninfected shoal members under predator attack
}

\author{
Nicolle Demandt ${ }^{1}$ - David Bierbach ${ }^{2,3} \cdot$ Ralf H. J. M. Kurvers ${ }^{4}$. Jens Krause ${ }^{2,3} \cdot$ Joachim Kurtz $^{1} \cdot$ Jörn P. Scharsack $^{1,5}$ (D)
}

Received: 4 May 2021 / Revised: 2 September 2021 / Accepted: 7 September 2021 / Published online: 4 October 2021

(c) The Author(s) 2021

\begin{abstract}
A key benefit of sociality is a reduction in predation risk. Cohesive group behaviour and rapid collective decision making are essential for reducing predation risk in groups. Parasite infection might reduce an individuals' grouping behaviours and thereby change the behaviour of the group as a whole. To investigate the relationship between parasite infection and grouping behaviours, we studied groups of three-spined sticklebacks, Gasterosteus aculeatus, varying the number of individuals experimentally infected with the cestode Schistocephalus solidus. We studied groups of six sticklebacks containing 0,2, 3, 4 or 6 infected individuals before and after a simulated bird attack. We predicted that infected individuals would have reduced shoaling and swimming speed and that the presence of infected individuals within a group would reduce group cohesion and speed. Uninfected fish increased shoaling and reduced swimming speed more than infected fish after the bird attack. In groups containing both infected and uninfected fish, the group behaviours were dominated by the more frequent character (uninfected versus infected). Interestingly, groups with equal numbers of uninfected and infected fish showed the least shoaling and had the lowest swimming speeds, suggesting that these groups failed to generate a majority and therefore displayed signs of indecisiveness by reducing their swimming speed the most. Our results provide evidence for a negative effect of infection on a group's shoaling behaviour, thereby potentially deteriorating collective decision making. The presence of infected individuals might thus have far-reaching consequences in natural populations under predation risk.
\end{abstract}

\section{Significance statement}

Parasite-infected individuals often show deviating group behaviours. This might reduce the anti-predator benefits of group living. However, it is unknown whether such deviations in group behaviour might influence the shoaling behaviour of uninfected group members and thereby the behaviour of the group as a whole. By experimentally infecting sticklebacks and investigating groups varying in infection rates, we show that infected sticklebacks differ in their shoaling behaviours from uninfected sticklebacks. Additionally, the presence of infected sticklebacks within the group affected the behaviour of uninfected shoal members. We show that shoals of infected fish are less cohesive and move slower compared to shoals of uninfected fish. Furthermore, we show that the infection rate of the shoal is crucial for how the group behaves.

Keywords Gasterosteus aculeatus $\cdot$ Schistocephalus solidus $\cdot$ Behavioural manipulation · Group behaviour · Shoaling behaviour $\cdot$ Predator attack

Communicated by T. C. M. Bakker.

This article is a contribution to the Topical Collection Sociality and Disease - Guest Editors: Rebeca Rosengaus, James Traniello, and Theo Bakker

Jörn P. Scharsack

joern.scharsack@thuenen.deAuthors and Affiliations

1 Institute for Evolution and Biodiversity, University of Münster, Hüfferstrasse 1, 48149 Münster, Germany

2 Leibniz-Institute of Freshwater Ecology and Inland Fisheries, Müggelseedamm 310, Berlin, Germany
3 Faculty of Life Sciences Humboldt, Universität Zu Berlin, Invalidenstrasse 42, 10115 Berlin, Germany

4 Center for Adaptive Rationality, Max Planck Institute for Human Development, Lentzeallee 94, 14195 Berlin, Germany

5 Present Address: Thünen Institute of Fisheries Ecology, Herwigstrasse 31, 27572 Bremerhaven, Germany 


\section{Introduction}

Sociality (i.e. the tendencies to form groups) is a common phenomenon in the animal kingdom (Pitcher 1986), with many animals spending at least part of their life as a member of a social group. One of the key advantages of living in social groups is a decrease in predation risk for the individual group members (Krebs and Davies 1993; Krause and Ruxton 2002). Group living can reduce the success rate of predator attacks, for example by increased prey vigilance (i.e. many eyes effect: Krause 1993a; Ioannou et al. 2012), confusion of predators (i.e. confusion effect: Godin 1986; Pitcher 1986; Ruxton et al. 2007; Ioannou et al. 2012) and reducing an individual's chance of being captured in a single predator attack (i.e. dilution effect: Foster and Treherne 1981). Local coordination is crucial to profit from the anti-predatory benefits of group living (Krause 1993b; Krebs and Davies 1993; Ruxton et al. 2007). However, a parasite infection might interfere with its host's ability to perform defensive and highly coordinated group behaviours (Barber et al. 2000). Such influences can be indirect, for example when infected individuals are actively avoided by uninfected conspecifics to reduce their probability of infection (Dugatkin et al. 1994; Barber et al. 2000; Ward et al. 2005; Tobler and Schlupp 2008). Parasites can also increase the conspicuousness of their host by altering colouration (Lafferty and Morris 1996; Seppälä et al. 2005). For example, the trematode Uvulifer $\mathrm{sp}$. causes black spots on the body surface of its host (western mosquitofish, Gambusia affinis), increasing its conspicuousness and potentially predation risk.

The influence of a parasite on its host's grouping behaviour can also occur more directly: the parasite can impair the sensorimotor control (Holmes and Zohar 1990; Muñoz et al. 2019) or anti-predator behaviour of its host (Barber et al. 2000). Especially, parasites with a complex life cycle (i.e. parasites that need multiple hosts) are known to influence the anti-predator behaviour of their host by reducing their flight response, to facilitate transmission to the next host (Barber et al. 2000; Poulin 2000). Finally, the presence of infected individuals in groups might also influence the grouping behaviour of uninfected group members (Beros et al. 2019), thereby potentially further decreasing the antipredatory benefits of grouping. Despite these possible farreaching influences of indirectly transmittable parasites, limited studies so far have been executed on how the presence of infected individuals and the parasite prevalence within a group might influence the group behaviour of both the uninfected and infected conspecifics.

Here, we experimentally infected three-spined stickleback, Gasterosteus aculeatus, with their cestode parasite, S. solidus (Hammerschmidt and Kurtz 2009; Barber and Scharsack 2010), to study the effect of infection on grouping behaviour. The three-spined stickleback is a small teleost fish occurring in marine and freshwater habitats all across the Northern Hemisphere (Wootton 1976). Sticklebacks can form shoals of up to hundreds of individuals in response to predators (Bell and Foster 1994; Poulin 1999).

The tapeworm $S$. solidus is a parasite with a three-host life cycle that infects sticklebacks as second intermediate hosts (Wootton 1976). S. solidus reproduces in the gut of its final host, a fish-eating bird. The bird, in turn, releases parasites' eggs in the water with its faeces. The parasite larvae hatch in the water and, next, infect their first intermediate host, a cyclopoid copepod (Hammerschmidt and Kurtz 2009). Infected copepods are then ingested by their obligatory second intermediate host, the three-spined stickleback (Barber and Scharsack 2010; Barber 2013). When $S$. solidus reaches sexual maturity and approximately $50 \mathrm{mg}$ of weight (Tierney et al. 1993), it starts disrupting the antipredator behaviour of infected sticklebacks (Bell and Foster 1994; Barber et al. 2000). Infected sticklebacks increase their risk-taking behaviour, by spending more time in open water and reducing their flight responses to a predator attack, thereby facilitating the parasite's transmission to its final host (Giles 1983, 1987; Milinski 1985; Barber et al. 2004; Quinn et al. 2012). Moreover, when satiated, infected sticklebacks decrease their social behaviour relative to uninfected conspecifics (Barber and Huntingford 1995). In two previous studies, we experimentally infected sticklebacks and studied their social behaviour, showing that uninfected sticklebacks adjusted their risk-taking behaviour to that of their infected shoal members when outnumbered (Demandt et al. 2018). Furthermore, we showed that infected sticklebacks hindered the spread of escape waves across stickleback groups (Demandt et al. 2020). However, the exact behavioural mechanisms of how infection impacts grouping behaviour remain unclear as these studies did not track individuals.

Here, we compared the shoaling behaviour of uninfected and experimentally infected sticklebacks, focusing both on how infection status impacts an individual's shoaling behaviours as well as how the infection rate of the shoal impacted the group's shoaling behaviour. The latter is especially relevant, as the prevalence of $S$. solidus-infected sticklebacks can vary considerably among populations (i.e. low prevalence of $1-3 \%$ or high prevalence of $>50 \%$ of the parasite; Arme and Owen 1967; Prieto et al. 2005; Kalbe et al. 2016; Weber et al. 2017). We tracked individuals in groups of six sticklebacks containing either $0,2,3,4$ or 6 infected individuals before and after an artificial bird attack. We predicted that shoals containing only uninfected sticklebacks would show higher cohesion than shoals containing only infected fish, especially after the bird strike. Furthermore, we expected that the shoal behaves according to the infection status of the majority. 


\section{Material and method}

\section{Experimental animals}

Laboratory-bred F1 offspring of three-spined sticklebacks and $S$. solidus caught in the brook Ibbenbürener Aa (Germany, 52 $\left.17^{\prime} 33.51^{\prime \prime} \mathrm{N}, 7^{\circ} 36^{\prime} 45.46^{\prime \prime} \mathrm{E}\right)$ were used. F1 stickleback families were obtained by in vitro fertilization and housed in family groups in 14-1 tanks (VewaTech, Germany) containing artificial plants for shelter. Sticklebacks were maintained in recirculating tap water at $18{ }^{\circ} \mathrm{C}$ with a light/dark cycle of $16 \mathrm{~h} / 8 \mathrm{~h}$ and fed daily ad libitum with frozen mosquito larvae (Chironomidae).

For parasite reproduction, the tapeworms were bred in vitro (Smyth 1954; Schärer and Wedekind 1999) in sizematched pairs to increase the probability of out-crossing (Lüscher and Milinski 2003). Parasite eggs were washed and stored at least 2 weeks at $4{ }^{\circ} \mathrm{C}$ to simulate winter conditions. The eggs were then incubated for 3 weeks at $20^{\circ} \mathrm{C}$ in the dark to enable coracidia (i.e. tapeworm larvae) development. The hatching of coracidia was subsequently initiated by illumination and eggs were kept in a light/dark cycle of $16 \mathrm{~h} / 8 \mathrm{~h}$ for 2 more days. Hatched coracidia were transferred to individual copepods in wells of 24-well plates with 2-ml tap water. At 14-day post-exposure, the copepods were checked for $S$. solidus infection with a microscope.

At approximately 6 months of age, the experimental sticklebacks $(n=332)$ —gathered from 12 families—were starved for 2 days and individually housed in jars with 400-ml tank water. The next day, sticklebacks were either offered S. solidusinfected copepods ( $n=222)$ or $S$. solidus-uninfected copepods (i.e. copepods not exposed to hatched coracidia; $n=110$ ). The ingestion of copepods was checked the next day by sieving the water from each jar, which confirmed that all copepods were ingested. Fish were then housed in groups of 11 individualsall from different families-in either all sham-exposed $(n=10$ groups) or all parasite-exposed ( $n=20$ groups) fish. After 69 days, the presence of $S$. solidus plerocercoids in the stickleback's body cavity was determined by detecting the swelling of the abdomen of the parasite-exposed sticklebacks (Barber 1997); 110 of the 222 parasite-exposed fish were classified as successfully infected. One day prior to the experiment, all fish received a small disc tag on their first spine (Webster and Laland 2009) for individual identification during the experiment. Each individual within the same holding tank received a unique disc colour.

\section{Experimental set-up}

To investigate the effect of parasite infection on shoaling behaviour before and after an artificial bird attack, five treatment groups were created; each group consisted of six sub-adult, non-reproductive sticklebacks $(\sim 50 \mathrm{~mm})$ and contained either 6, 4, 3, 2 or 0 uninfected fish (and 0, 2, 3, 4 or 6 infected fish, respectively). All treatment groups were replicated seven times resulting in a total of 105 uninfected and 105 infected fish. Fish were randomly assigned to a group with the constraint that fish within a group did not come from the same holding tank (to standardize familiarity), nor had the same disc colour. To minimize observer bias, blinded methods were used to analyse the behavioural data.

The experimental tank $(80 \times 35 \times 40 \mathrm{~cm})$ for the shoaling experiment was filled with $16-\mathrm{cm}$ white gravel. A thin white acrylic glass plate was placed on the gravel to increase the contrast between the fish and the background and reduce the walls' shadows. The tank was filled with $7-\mathrm{cm}$ water to facilitate 2D tracking. A cardboard silhouette of a kingfisher in flight (i.e. wings extended outwards from the body) was placed next to the tank; it was out of sight from the fish when not triggered. All of the tank's walls were covered with nonreflective white acrylic glass. At each short side, one tube was attached for draining and filling the tank. Above the tank and artificial bird, behind a grey Plexiglas plate with a small hole in it, a Logitech ${ }^{\circledR}$ HD pro C910 webcam was placed to record the trials with $30 \mathrm{fps}$. The experimental set-up was placed in a shelf $(100 \times 50 \times 200 \mathrm{~cm})$. Two tube lights $(\mathrm{cool}$ daylight $39 \mathrm{~W} /$ between 865 and $2850 \mathrm{~lm}$ max) were placed at either long side of the set-up, to minimize the light reflection inside the tank. The shelf itself was shielded by black cloths; the operator could manually trigger the bird attack from behind the cloth.

\section{Test procedure and behavioural observation}

During the experiment, all fish were fed ad libitum with chironomid larvae in the morning to standardize satiation levels (see also Barber and Huntingford 1995). Before the start of a trial, the six assigned sticklebacks were dip netted from their respective holding tanks and moved to the experimental room in a bucket. All sticklebacks were then gathered in a small cup and simultaneously released into the experimental tank. After 5 min of acclimatization, a 5-min observation period started. Next, an artificial bird flying over the experimental tank was triggered. This was done when all fish were facing the corner where the artificial bird was hidden, and took maximally $60 \mathrm{~s}$ to occur, to ensure the detection of the threat by all fish. The observations continued for another $5 \mathrm{~min}$ after the bird attack. Fish were then returned to their original holding tanks. The experimental tank was cleaned and refilled with new water between trials. 


\section{Tracking and data collection}

To acquire individual movement data, the two 5-min recordings before and after the bird attack were tracked with Noldus Ethovision XT 14 (Noldus et al. 2001) at a frame rate of 15 fps. A combination of colour and shape tracking was used as some of the disc tags were difficult to track by colour alone. All tracked trajectories were visually checked for inconsistencies and errors were manually corrected. Thus, we conceived individual tracks of all individuals within a group. All coordinates were converted from pixel to centimetre.

\section{Data analysis shoaling behaviour}

\section{Individual-level measurements}

We determined for each fish its (i) nearest neighbour distance (NND) and (ii) swimming speed from tracking data (see Appendix for details). We did this separately for the period before and after the bird attack. For the statistical analysis, we used the median of these measurements as the median is more robust than the mean (Galton 1907). All measurements were divided by an individual's body length to control for possible body size effects. Additionally, we determined for each fish its proportion of time mobile (defined as moving faster than half a body length per second; Jolles et al. 2020).

\section{Group-level measurements}

We determined for each group the (i) median speed of the group centre, (ii) maximum speed of the group centre and (iii) mean inter-individual distance (IID) (see Appendix for details). We did this separately for the period before and after the bird attack. For all calculations, MATLAB 2017b (MATLAB 2017) was used.

\section{Statistical analysis}

We used R 4.0.2. (R Core Team 2018) for statistical analysis and the function ggplot (package 'ggplot2'; Wickham 2016) for graphs.

\section{Individual-level measurements}

The individual-level measures were analysed in four subsets: (a) 'pure groups', comprising the groups with all shamexposed and all infected individuals, (b) sham-exposed individuals, to test whether sham-exposed individuals were affected by group composition, (c) infected individuals, to test whether infected individuals were affected by group composition and (d) 'mixed groups', groups containing both sham-exposed and infected individuals to test for an interaction between individual and group infection status.
To analyse the effect of treatment and bird attack (i.e. before or after bird attack) on shoaling parameters (i.e. NND, swimming speed and proportion of time mobile), linear mixed models (LMMs) were used. In case of homoscedasticity, the function lmer (package 'Ime4'; Bates et al. 2015) was used; in case of heteroscedasticity, lme (package 'nlme'; Pinheiro et al. 2020) was used (see Table S1 for model details). Visual inspection of the data indicated a gamma distribution for the NND of uninfected individuals. Therefore, to analyse the effect of treatment and bird attack on the NND of uninfected individuals, generalized linear mixed models (GLMMs) were used with the function glmer (package 'Ime4') and a gamma distribution with inverse link function. The control function glmerControl(optimizer="bobyqa",op $\mathrm{tCtrl}=$ list $(\operatorname{maxfun}=2 \mathrm{e} 5))$ was used to facilitate convergence (see Table $\mathrm{S} 1$ for model details).

In all models, treatment, bird attack and its two-way interaction were fitted as fixed effects. In the models for the 'mixed groups', individual infection status (uninfected versus infected) and all additional two- and three-way interactions were also included. Fish ID nested in group ID was included as a random effect in all models.

For all GLMMs, and for the LMMs after refitting to maximum likelihood, the significance of the fixed effects was determined using likelihood ratio tests (LRT) and a stepwise backward elimination, with the drop1 function (package 'stats'; R Core Team 2018) and the AIC values, to obtain the minimum adequate models (MaM). After determining the MaM, all LMMs were refitted to restricted maximum likelihood, and the residuals of all models were visually (function qqPlot, package 'car', Zuur et al. 2009; and function plot, package 'stats' R Core Team 2018) and statistically inspected for normality (function ad.test, package 'nortest'; Gross and Ligges 2015) and homogeneity (function leveneTest, package 'car'). For the analysis of the NND, the analysis of the swimming speed of the infected individuals' and the analysis of the proportion of time mobile for the uninfected individuals', and the pure groups', abnormalities were found. After checking the residuals, post hoc tests were performed using the function emmeans (package 'emmeans'; Lenth 2020) with a Benjamini-Hochberg correction to investigate subgroup differences.

\section{Group-level measurements}

To determine the effect of treatment and bird attack on the (i) median group speed, LMMs with the function lme were used, (ii) maximum group speed LMMs with the lmer function were used and (iii) on the mean IID of each group, GLMMs with the glmer function were used. For the latter, a gamma distribution with inverse link function and 
the control function glmerControl(optimizer="bobyqa", optCtrl=list $(\max f u n=2 \mathrm{e} 5))$ were used. Treatment, bird attack and their two-way interaction were fitted as fixed effects, and Group ID as random effect in all models (see Table $\mathbf{S} 2$ for model details).

For all GLMMs, and for the LMMs after refitting to maximum likelihood, the significance of the fixed effects was determined using likelihood ratio tests (LRT) and a stepwise backward elimination, with the drop1 function (package 'stats') and the AIC values, to obtain the minimum adequate models (MaM). After determining the MaM, all LMMs were first refitted to restricted maximum likelihood, and the residuals of all models were visually (Zuur et al. 2009) (functions qqPlot, package 'car' and plot, package 'stats') and statistically inspected for normality (function ad.test, package 'nortest') and homogeneity (function leveneTest, package 'car'). Only for the analysis of the nearest neighbour distances of the all infected and mixed groups, abnormalities were found. No heteroscedasticity was found. After checking the residuals, post hoc tests were performed using the function emmeans (package 'emmeans') with a Benjamini-Hochberg correction to investigate subgroup differences.

\section{Results}

\section{Effects of parasite infection on individual shoaling behaviours}

\section{Shoaling behaviour of 'pure' groups}

We first investigated if sticklebacks in the 'pure' groupscomprising of only uninfected (6u) and only infected fish (6i) - differed in their shoaling behaviours before and after the bird attack. Comparing the nearest neighbour distances (NND) of only uninfected and only infected fish revealed no significant interaction between treatment and bird attack (LRT $=0.47, \mathrm{df}=1, p=0.49$ ). Uninfected fish had a lower NND than infected fish $(\mathrm{LRT}=5.20, \mathrm{df}=1, p=0.023$; Fig. 1a), and fish decreased their NND after the bird attack $(\mathrm{LRT}=16.66, \mathrm{df}=1, p<0.001$; Fig. 1a, b).

There was a tendency for an interaction between treatment and bird attack on the swimming speed (LRT $=3.04$, $\mathrm{df}=1, p=0.081$; Fig. 1c, d). Fish swam slower after the bird attack $(\mathrm{LRT}=65.41, \mathrm{df}=1, p<0.001)$ and uninfected fish tended to swim faster compared to infected fish (LRT $=2.78$, $\mathrm{df}=1, p=0.095$; Fig. 1c).

There was no significant interaction between treatment and bird attack on the proportion of time mobile (LRT $=0.51, \mathrm{df}=1, p=0.47$; Fig. 1e, f). Uninfected and infected fish did not differ in their mobility (LRT $=0.80$, $\mathrm{df}=1, p=0.37$ ) and fish reduced their mobility after the bird attack $(\mathrm{LRT}=25.94, \mathrm{df}=1, p<0.001)$.

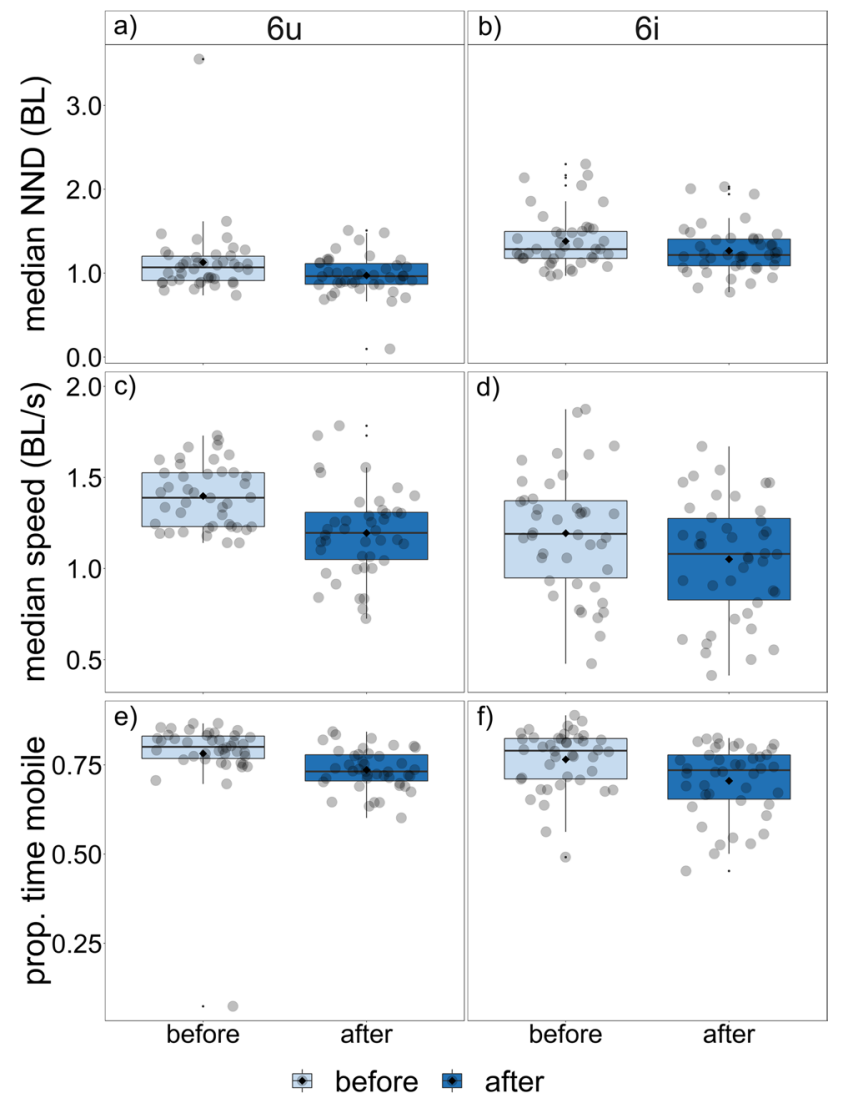

Fig. 1 Individual grouping measurements of sticklebacks from the only uninfected (6u) and only infected (6i) groups before (light blue) and after (dark blue) an artificial bird attack. a-b Median nearest neighbour distance (BL), c-d median swimming speed (BL/s) and e-f proportion of time mobile. The edges of the box plots indicate the first and third quartiles; the solid lines the median, the diamonds the mean, the whiskers the highest and lowest values within 1.5-fold of the inter-quartile range and the transparent dots represent each individual (grey) of seven replicates each ( $n=42$ per treatment)

\section{Shoaling behaviour of uninfected sticklebacks}

To investigate whether the shoaling behaviour of uninfected sticklebacks was influenced by the group composition, we compared the shoaling behaviour of uninfected sticklebacks between treatments before and after the bird attack.

Independent of bird attack, the NND of uninfected sticklebacks was influenced by treatment (LRT $=8.55, \mathrm{df}=3$, $p=0.04$; Fig. 2a-d; Table S3a). Post hoc comparisons revealed that uninfected fish from treatment $6 u$ always had a lower NND compared to uninfected fish from treatment $3 \mathrm{u} / 3 \mathrm{i}(z=2.8, p=0.03)$ and $4 \mathrm{u} / 2 \mathrm{i}(z=2.63, p=0.03)$, while no differences in NND were found between the other treatments (all $p>0.22$; Table S4a). There was a significant interaction between treatment and bird attack on NND $($ LRT $=18.95, \mathrm{df}=3, p<0.001$; Fig. 2a-d; Table S3a). Post hoc comparisons revealed that before the bird attack, uninfected fish in treatment $6 \mathrm{u}$ had a slightly lower NND 


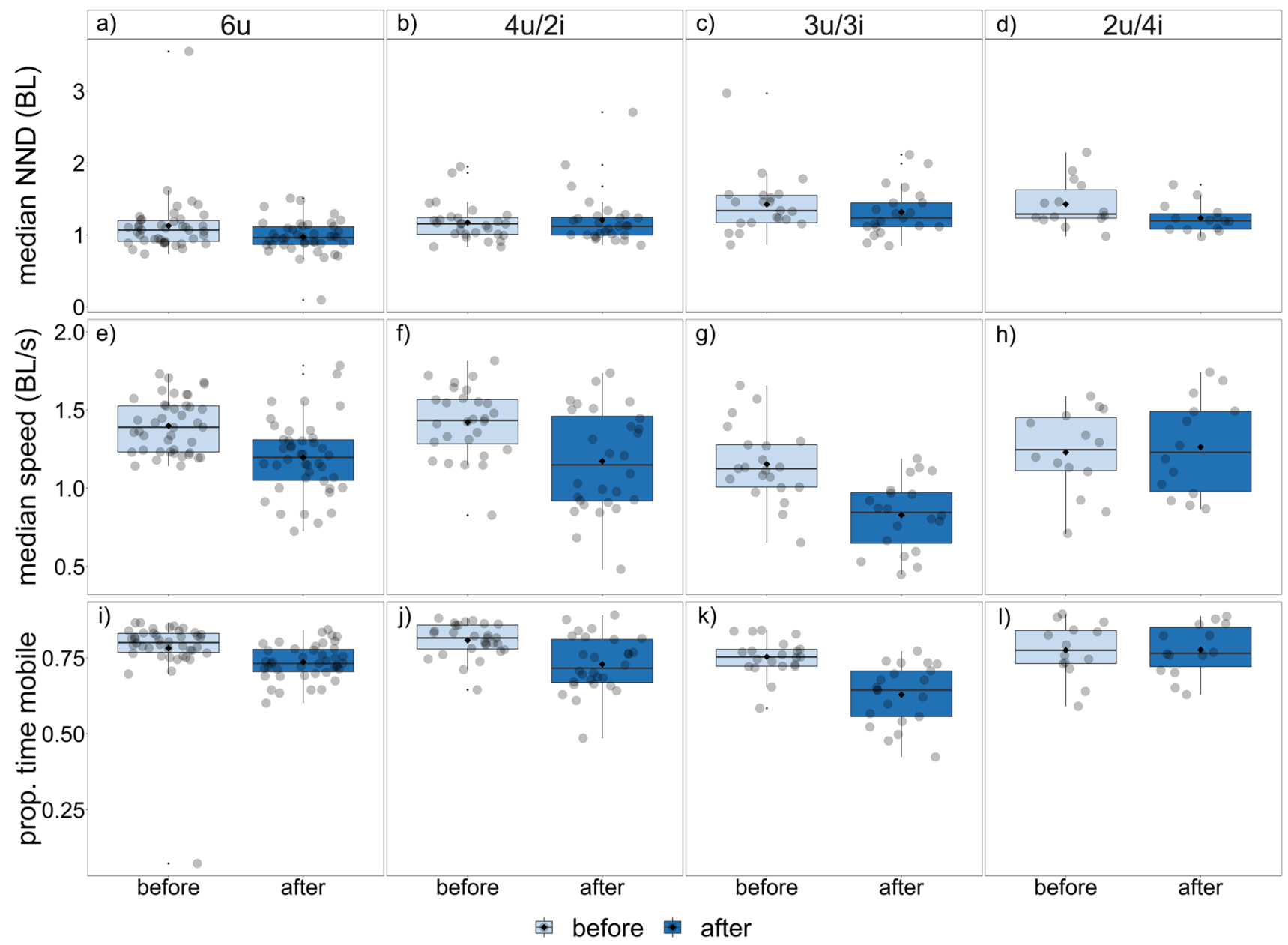

Fig. 2 Individual grouping measurements of only the uninfected sticklebacks per treatment before (light blue) and after (dark blue) an artificial bird attack. Treatment groups comprised of uninfected (u) and infected (i) sticklebacks in the combinations: $6 u(a, e, i), 4 u / 2 i$ (b, f, j), 3u/3i (c, g, k) and 2u/4i (d, h, l). a-d Median NND (BL), e-h median swimming speed (BL/s) and $\mathbf{j}-\mathbf{l}$ proportion of time mobile. The edges of the box plots indicate the first and third quartiles; the solid lines the median, the diamonds the mean, the whiskers the highest and lowest values within 1.5-fold of the inter-quartile range and the transparent dots represent each uninfected stickleback compared to uninfected fish in the treatments $3 \mathrm{u} / 3 \mathrm{i}$ $(z=2.28, p=0.07$; Fig. $2 \mathrm{a}, \mathrm{d})$ and $2 \mathrm{u} / 4 \mathrm{i}(z=2.44, p=0.07$; Fig. 2a, c; Table S5a). After the bird attack, uninfected fish in treatment $6 \mathrm{u}$ had a lower NND compared to uninfected fish in the other treatments (all $p<0.02$; Fig. 2a-d), while no differences in NND were found for the uninfected fish in the other treatments (all $p>0.73$; Fig. 2b-d; Table S5a). Uninfected fish in treatments $6 \mathrm{u}$ and $2 \mathrm{u} / 4 \mathrm{i}$ reduced the NND after the bird attack (both $p<0.02$ ), while the bird attack did not influence the NND of the uninfected fish in treatment $4 \mathrm{u} / 2 \mathrm{i}$ and $3 \mathrm{u} / 3 \mathrm{i}$ (both $p>0.12$; Table S5a).

In general, the median swimming speed of uninfected sticklebacks was influenced by treatment (LRT $=11.54$, df $=3, p=0.009$; Fig. 2e-h; Table S3a). Post hoc comparisons revealed that uninfected fish from treatment $3 \mathrm{u} / 3 \mathrm{i}$ always had lower median swimming speed compared to the uninfected fish from the other treatments (all $p<0.027$;
Table S4b), while no general differences were found for the uninfected fish in the other treatments (all $p>0.73$; Table S4b). There was a significant interaction between treatment and bird attack on the median swimming speed $(\mathrm{LRT}=19.44, \mathrm{df}=3, p<0.001$; Fig. 2e-h; Table S3b). Post hoc comparisons revealed that before the bird attack, uninfected fish in treatment $6 \mathrm{u}$ and $4 \mathrm{u} / 2 \mathrm{i}$ tended to swim faster compared to uninfected fish in treatment $3 \mathrm{u} / 3 \mathrm{i}$ (both $p<0.069$; Fig. 2e-g; Table S5b). After the bird attack, uninfected fish in treatment $3 \mathrm{u} / 3 \mathrm{i}$ swam significantly slower than the uninfected fish in the other treatments (all $p<0.004$; Table S5b), whereas the uninfected fish from the other treatments did not differ in swimming speed (all $p>0.62$; Fig. 2e-h; Table S5b). Furthermore, all uninfected fishexcept for the ones in treatment $2 \mathrm{u} / 4 \mathrm{i}(t=0.58, p=0.56$; Fig. 2h) - decreased their swimming speed after the bird strike (all $p<0.001$; Table S5b). 
Finally, independent of bird attack, the proportion of time mobile was significantly influenced by treatment (LRT = 12.567, df =3, $p=0.006$; Fig. 2i-1; Table S3c). Post hoc comparisons revealed that uninfected fish from treatment $3 \mathrm{u} / 3 \mathrm{i}$ spent less time mobile compared to uninfected fish from the other treatments (all $p<0.022$; Table S4c), while no general differences existed among uninfected fish from the other treatments (all $p>0.37$; Table S4c). There was a significant interaction between treatment and bird attack on the proportion of time mobile (LRT $=21.23, \mathrm{df}=3$, $p<0.001$; Fig. 2i-l; Table S3c). Post hoc tests revealed that before the bird attack, there were no treatment differences in the proportion of time uninfected fish were mobile (all $p>0.70$; Table S5c). After the bird attack, uninfected fish in treatment $3 \mathrm{u} / 3 \mathrm{i}$ spent less time mobile compared to the uninfected fish in the other treatments (all $p<0.01$ ), whereas the uninfected fish from the other treatments did not differ in their mobility (all $p>0.26$; Table S5c). All uninfected fishexcept for the ones in treatment $2 \mathrm{u} / 4 \mathrm{i}(t=-0.08, p=0.94$; Fig. 2l)—decreased their mobility after the bird strike (all $p<0.005$; Table S5c).

Tables S6-S7 show the results for the shoaling behaviour of infected fish. In short, a significant interaction between treatment and bird attack was found on the NND and proportion of time mobile of infected fish (both $p<0.030$; Fig. S1a-d, i-1; Table S6a, c). Before the bird attack, there were no treatment differences in the NND and proportion of time (all $p>0.40$; Table S7). After the bird attack, infected fish in treatment $3 \mathrm{u} / 3 \mathrm{i}$ tended to spent less time mobile compared to the infected fish in treatment $2 \mathrm{u} / 4 \mathrm{i}(t=-2.69$, $p=0.077$; Fig. S1i, j; Table S7b). All infected fish-except for the ones in treatment $4 \mathrm{u} / 2 \mathrm{i}(t=1.82, p=0.07$; Fig. S1a; Table S7a) - reduced their NND after the bird attack (all $p<0.002)$. Furthermore, infected fish reduced their mobility (all $p<0.009$; Table S7b) and swimming speed (LRT $=54.91$, $\mathrm{df}=1, p<0.001$; Table S6b) after the bird attack.

Finally, in the mixed groups, uninfected and infected fish did not significantly differ in their NND, median swimming speed or the proportion of time mobile, neither before nor after the bird attack (all $p>0.065$; Table S7).

\section{Effects of parasite infection on shoaling behaviours of the group as a whole}

Independent of the bird attack, the median group speed was significantly influence by treatment (LRT $=13.419$, $\mathrm{df}=4, p=0.009$; Fig. 3a-e; Table S9a). Groups from treatment $6 \mathrm{u}$ and $4 \mathrm{u} / 2 \mathrm{i}$ had a significantly higher median group speed compared to groups from treatment $3 \mathrm{u} / 3 \mathrm{i}$ (both $p<0.029$; Table $\mathrm{S} 10$ ) and a slightly higher median group speed compared to groups from treatment $6 \mathrm{i}$ (both $p<0.09$; Table S10). Additionally, groups from treatment $2 \mathrm{u} / 4 \mathrm{i}$ tended to have a higher median group speed compared to groups from treatment $3 \mathrm{u} / 3 \mathrm{i}(\mathrm{t}=-2.09$, $p=0.09$ ). Furthermore, there was a significant interaction between treatment and bird attack on the median group speed (LRT $=10.50, \mathrm{df}=4, p=0.033$; Fig. 3a-e; Table S9a). Post hoc comparisons revealed that before the bird attack, groups from treatment $6 \mathrm{u}$ and $4 \mathrm{u} / 2 \mathrm{i}$ had a higher median group speed compared to groups from treatment $3 \mathrm{u} / 3 \mathrm{i}$ and $6 \mathrm{i}($ all $p<0.035)$. There were no differences in median group speed between the other treatments (all $p>0.13$; Fig. 3a-e; Table S11a). After the bird attack, groups from treatment $3 \mathrm{u} / 3 \mathrm{i}$ had a significantly lower median group speed compared to groups from other treatments (all $p<0.040$ ). The groups from the other treatments did not differ in median group speed (all $p>0.27$; Fig. 3a-e; Table S11a). This suggests that groups from treatment $3 \mathrm{u} / 3 \mathrm{i}$ had difficulties to synchronize their swimming speed. After the bird attack, groups from treatment $6 \mathrm{u}, 4 \mathrm{u} 2 \mathrm{i}$ and $3 \mathrm{u} / 3 \mathrm{i}$ reduced their median group speed (all $p<0.030$; Fig. 3a-c), whereas the groups from treatment $2 \mathrm{u} / 4 \mathrm{i}$ and $6 \mathrm{i}$ did not decrease their median group speed (both $p>0.33$; Fig. 3d, e; Table S11a).

Comparing the maximum swimming speed of the different treatment groups revealed no significant interaction between treatment and bird attack $(\mathrm{LRT}=2.88, \mathrm{df}=4$, $p=0.58$; Fig. $3 \mathrm{f}-\mathrm{j}$; Table S9b). However, the maximum swimming speed of each group was influenced by the treatment ( $\mathrm{LRT}=11.92, \mathrm{df}=4, p=0.02$; Table S9b). Post hoc comparisons revealed that the maximum speed of groups from treatment $6 \mathrm{u}$ was higher compared to groups from treatment $3 \mathrm{u} / 3 \mathrm{i}(t=3.08, p=0.04$; Fig. 3f, h). No differences in maximum speed were found between the groups from the other treatments (all $p>0.11$; Table S11b). Furthermore, the maximum swimming speed tended to be slightly higher after the bird attack $(\mathrm{LRT}=3.52, \mathrm{df}=1, p=0.06)$.

Additionally, there was no significant interaction between treatment and bird attack on the mean inter-individual distance (IID) within a group (LRT $=4.83, \mathrm{df}=4, p=0.31$; Fig. 3k-0; Table S9c). No difference in IID was observed between the different treatments $(\mathrm{LRT}=2.82, \mathrm{df}=4$, $p=0.59)$. However, all groups reduced their IID after the bird attack $(\mathrm{LRT}=25.09, \mathrm{df}=1, p<0.001)$.

\section{Discussion}

Reduction in predation risk is one of the key advantages of group living (Krebs and Davies 1993; Krause and Ruxton 2002). Shoaling behaviours (i.e. low nearest neighbour distances and high inter-individual coordination) are essential for the reduction of predation risk of individual shoal members. Parasites that manipulate their host's behaviour might interfere with their host's ability to shoal. Here, we tested if experimentally infected sticklebacks differed in their shoaling 


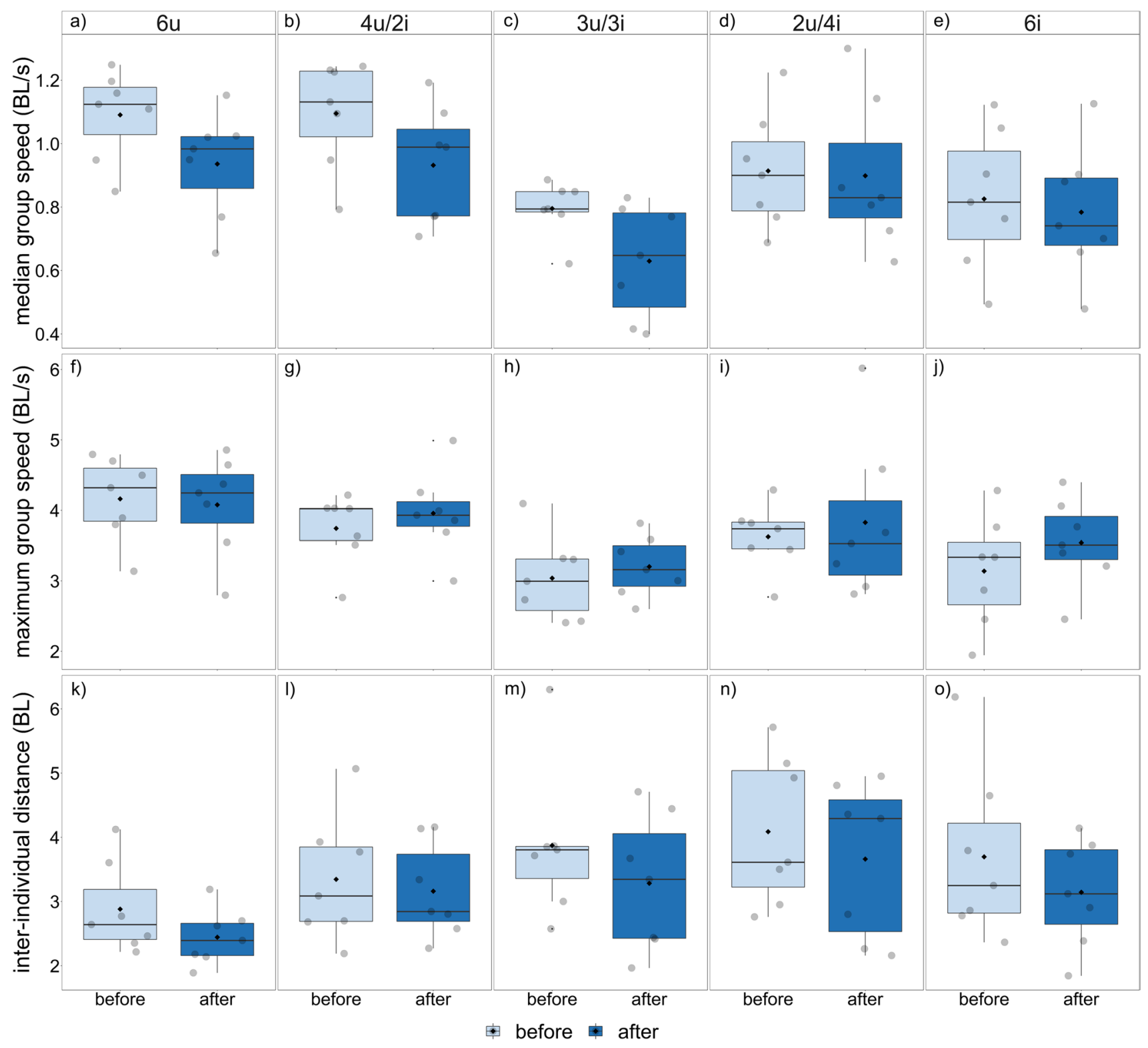

Fig. 3 Grouping measurements of all groups before (light blue) and after (dark blue) an artificial bird attack. Treatment groups comprised of uninfected (u) and infected (i) sticklebacks in the combinations: $6 \mathrm{u}$ (a), 4u/2i (b), 3u/3i (c), 2u/4i (d) and 6i (e). a-e Median group speed $(\mathrm{BL} / \mathrm{s}), \mathbf{f}-\mathbf{j}$ maximum group speed $(\mathrm{BL} / \mathrm{s})$ and $\mathbf{k}-\mathbf{o}$ inter-individual

behaviour and whether the presence of such individuals changed the shoaling behaviour of uninfected shoal mates and that of their group as a whole.

Groups consisting of only uninfected individuals formed more cohesive (i.e. lower NND) and slightly faster swimming groups than groups consisting of only infected individuals. In groups harbouring both uninfected and infected individuals, the character (uninfected vs. infected) that outnumbered the other dominated the shoal's behaviour before the bird attack. In a quorum decision like manner (Ward distances (BL). The edges of the box plots indicate the first and third quartiles; the solid lines the median, the diamonds the mean, the whiskers the highest and lowest values within 1.5 -fold of the interquartile range and the transparent dots represent each group

et al. 2008, 2012; Sumpter and Pratt 2009), all individuals of the shoal (no matter if infected or uninfected) tended to adopt the majority's behaviour. After simulated bird attack, all groups became more cohesive and stationary, but this effect was stronger in groups where uninfected individuals outnumbered infected individuals. Groups with more infected individuals responded less to the bird attack, which might be explained by the parasite-induced behavioural manipulation that makes stickleback hosts more prone to bird predation (Giles 1983, 1987; Milinski 1985; Barber et al. 2004; Quinn 
et al. 2012). Most strikingly, upon bird attack, groups that comprised of equal numbers of uninfected and infected fish displayed the lowest cohesion and swimming speed. This result suggests that these groups failed to generate a majority and therefore displayed signs of indecisiveness by reducing their swimming activity the most.

Parasite-infected individuals frequently display altered shoaling behaviours (Barber et al. 2000; Ward et al. 2002; Jolles et al. 2020). In the present study, we illustrated that the changes in the shoaling behaviour of S. solidus-infected sticklebacks influenced the shoaling behaviour of uninfected conspecifics and that of the group as a whole.. We propose three non-mutually exclusive explanations for this change in shoaling behaviour. First, the reduced shoaling behaviour of infected fish may be because of an increased drag and reduced flexibility due to their distended abdomen (Milinski 1985; Barber 1997; Barber et al. 2000; Ghalambor et al. 2004), especially, since a higher drag leads to a higher cost when swimming fast (Videler 1993). Second, the infection might change the trade-off between foraging and sociality. Infected fish could be in a permanent state of hunger, which could increase their motivation to search for food as compared to uninfected fish. The stomach of heavily S. solidusinfected individuals can be extensively compressed by to the presence of the growing parasite in the abdominal cavity (Milinski 1985), reducing their capacity to ingest food. This may force them to consume prey more frequently than uninfected fish. As the fish in our experiments were fed ad libitum before the start of the experiments, the reduced capacity to ingest food at one time point might have led to increasing differences in hunger levels between the uninfected and infected fish towards the end of the day. Such differences in hunger levels might have affected the hosts' behaviours (Hafer and Benesh 2015; Hafer and Milinski 2016). Furthermore, the parasite also causes a major energetic drain on the energy requirements of the host (Milinski 1990; Barber and Scharsack 2010; Hafer and Milinski 2016). Such high energetic requirement is also expected to change the behaviour from grouping towards foraging (Pascoe and Mattey 1977; Milinski 1990; Pitcher and Parrish 1993; Barber and Scharsack 2010; Lafferty and Shaw 2013). Lastly, infected fish have a higher resting metabolism than uninfected fish, increasing their swimming costs (Lester 1971), which may make them less prone to swim fast. Third, S. solidus manipulates the behaviour of its host to enhance transmission to the bird (Giles 1983, 1987; Milinski 1985; Barber and Huntingford 1995; Barber et al. 2004). The observed reduction in swimming speed and shoaling of infected fish might benefit the parasite by further increasing its chances of transmission to the final host. Therefore, our observed effects might also represent active parasite-induced behavioural manipulation.

Interestingly, the shoaling behaviour of the uninfected and infected fish within one group did not differ. A possible explanation might be that individuals within a group-independent of infection status-adjust their behaviours conform to the group. Group conformity increases with group size, thereby increasing the benefits of risk dilution and confusion effects (Pitcher 1986; Ioannou et al. 2012). On the short term, conforming to the social group seems favourable for both uninfected and infected fish. However, on the long run, such conformity may be too costly for infected fish as they suffer from increased energy expenditure when swimming (Lester 1971; Barber and Svensson 2003). Especially, in our flow-less lab situations, infected individuals might be able to conform to the social groups swimming speed more easily and at relatively low costs.

In natural environments, however, sticklebacks can live in streams with high flow rates (Wootton 1976). Therefore, it would be interesting to test the influence of parasiteinfected individuals on shoals in environments with rapid flows or in a lab experiment where the flow is increased experimentally. Possibly, infected individuals will be exhausted more rapidly under such conditions, preventing them from keeping up with their shoal mates, which could lead to positive assortment based on parasite burden. Another possibility is that infected fish select shoal positions that provide higher energetic benefits (i.e. trailing positions within a shoal (Herskin and Steffensen 1998; Krause and Ruxton 2002; Johansen et al. 2010; Marras et al. 2015)), even though these positions could also have disadvantages, such as reduced foraging success (Krause 1993a; Krause and Ruxton 2002).

After experiencing predator cues, fish shoals tend to become more compact and synchronized (Bode et al. 2010; Schaerf et al. 2017) — which was also the case in our experiments but was attenuated by the presence of parasite-infected individuals. In the evenly mixed groups of the present study, the escape behaviour of half of the shoal members was manipulated by a parasite that makes its stickleback host bolder (Milinski 1985; Demandt et al. 2018) and less responsive to danger signals from shoaling conspecifics (Demandt et al. 2020). When the uninfected half of the sticklebacks received a trigger for their flight instinct, an internal conflict occurred as they observed that the infected half of the shoal did not show a strong fright response following the predator attack. Consequently, the group cohesion was disrupted due to the occurrence of confusion within the group caused by the competing response tendencies of the uninfected and infected fish. As a result, it is possible that the group needed longer to reach a decision (i.e. which direction to move to). During a natural predator attack, such indecisiveness might be fatal and elevate the predation risk if the individuals cannot coordinate quickly. It would be interesting to investigate whether such disruption of group cohesion only occurs in small groups (like in our experiments and Jolles et al. 2020) or also in bigger groups that might split up into multiple subgroups. 
An important question in this context is how predators would respond to a group that displays indecisiveness and then splits. A possibility is that the predator gets confused, as has been described for shoals of fish that open up to swim around predators (Partridge 1982). However, the latter is commonly conducted in a controlled manner and not initiated with a phase of indecisiveness or confusion of the shoal members themselves.

Previous studies have shown that the susceptibility to parasite infections might differ among individuals and might be related to the sex and/or physical conditions of an individual (Wilson et al. 1993; Zuk and McKean 1996; Koolhaas et al. 1999; Arnold et al. 2003; Ferrari et al. 2007; Barber and Dingemanse 2010; Boyer et al. 2010). For example, shoals of only male or only female guppies, $P$. reticulate, differed in their infection risk with the transmittable parasite Gyrodactylus turnibulli (Richards et al. 2010). Male sticklebacks were found to harbour more Glugea anomala cysts compared to adult females (Arnold et al. 2003). Besides a sex difference, individuals also vary in their investment in their immune system with some individuals investing more energy in their innate compared to their adaptive immune system and vice versa (Koolhaas et al. 1999). Such investment is highly relevant, as it has been shown that the cellular innate immune response of an individual stickleback plays an important role for the clearance of an early $S$. solidus infection (Scharsack et al. 2007; Barber and Scharsack 2010). It is possible that our infected sticklebacks were a non-random subset of the exposed group, which might already have differed in some of the shoaling properties before infections. It is therefore important for future studies to also include a control group consisting of exposed but uninfected sticklebacks to assure that all observed shoaling differences were directly caused by the parasite infection.

Our results offer some intriguing aspects of shoaling with infected individuals. When uninfected fish outnumber the infected fish, the group as a whole seems to display similar shoaling behaviours as shoals that do not contain any infected fish. Therefore, the presence of some infected individuals within a group might not reduce the anti-predator benefits of shoaling. Most strikingly, when infected fish occurred in numbers equal to uninfected ones, group cohesion was dramatically reduced after a predation stimulus. The dramatic alteration of the groups' anti-predator behaviour when infected individuals take up more than $50 \%$ of the group has important implications for collective decision making. Therefore, it has to be expected that some selection for the avoidance of grouping with infected individuals by healthy individuals should occur. However, so far, no evidence for this exists and in the wild uninfected individuals frequently co-occur with infected individuals when the parasites do not transfer horizontally (Scharf et al. 2012; Weber et al. 2017; Gracia et al. 2018). This suggests that the benefits of grouping with infected individuals might outweigh the costs under certain environmental conditions (Scharf et al. 2012). Therefore, future research should try to identify under which conditions the costs of grouping with infected individuals might outweigh the benefits. In summary, the alterations in shoaling behaviour might have far-reaching consequences for individuals grouping with infected individuals, especially in predator rich environments and when the ratios of uninfected and infected individuals are balanced.

Supplementary Information The online version contains supplementary material available at https://doi.org/10.1007/s00265-021-03080-7.

Acknowledgements We thank Kathrin Brüggemann, Georg Plenge, Luis Garcia Rodriguez and further members of the workshop of the faculty of Biology for their technical assistance. We further thank Marit Praets, Sophie Bienert and Leonie Bley for help with fish husbandry. We thank Christopher Schutz for helping with the tracking of the videos. We furthermore thank two anonymous referees for their comments on an earlier version of the manuscript.

Author contributions $\mathrm{ND}, \mathrm{JKu}$ and JS established the experimental set-up. ND performed the behavioural observations of the sticklebacks. ND and DB tracked the videos with Ethovision. RK, DB, JKr and ND discussed the analysis of the tracking data. ND prepared the statistical analysis and wrote the first draft of the manuscript. All authors commented on previous versions of the manuscript. All authors read and approved the final manuscript.

Funding Open Access funding enabled and organized by Projekt DEAL. The project was supported by a DAAD stipend (No. 91557716) to ND and by Germany's Excellence Strategy (EXC 2002/1 'Science of Intelligence', project number 390523135 to JKr).

Data availability All data analysed during this study are included in the supplementary information files.

\section{Declarations}

Competing interests The authors declare no competing interests.

Ethical approval All animal experimental procedures were executed in accordance with the EU Directive 2010/63/EU for animal experiments and all applicable international, national and/or institutional guidelines for the use of animals in experiments were followed. Sticklebacks were maintained and treated with approval of the local veterinary and animal welfare authorities under the Project Number: 84-02.04.2014.A368.

Open Access This article is licensed under a Creative Commons Attribution 4.0 International License, which permits use, sharing, adaptation, distribution and reproduction in any medium or format, as long as you give appropriate credit to the original author(s) and the source, provide a link to the Creative Commons licence, and indicate if changes were made. The images or other third party material in this article are included in the article's Creative Commons licence, unless indicated otherwise in a credit line to the material. If material is not included in the article's Creative Commons licence and your intended use is not permitted by statutory regulation or exceeds the permitted use, you will need to obtain permission directly from the copyright holder. To view a copy of this licence, visit http://creativecommons.org/licenses/by/4.0/. 


\section{References}

Arme C, Owen RW (1967) Infections of the three-spined stickleback, Gasterosteus aculeatus L., with the plerocercoid larvae of Schistocephalus solidus (Müller, 1776), with special reference to pathological effects. Parasitology 57:301-314. https://doi. org/10.1017/S0031182000072103

Arnold KE, Adam A, Orr KJ, Griffiths R, Barber I (2003) Sexspecific survival and parasitism in three-spined sticklebacks: seasonal patterns revealed by molecular analysis. J Fish Biol 63:1046-1050. https://doi.org/10.1046/j.1095-8649.2003. 00195. $\mathrm{x}$

Barber I (1997) A non-invasive morphometric technique for estimating cestode plerocercoid burden in small freshwater fish. J Fish Biol 51:654-658. https://doi.org/10.1111/j.1095-8649.1997.tb01521.x

Barber I (2013) Sticklebacks as model hosts in ecological and evolutionary parasitology. Trends Parasitol 29:556-566. https://doi.org/ 10.1016/j.pt.2013.09.004

Barber I, Dingemanse NJ (2010) Parasitism and the evolutionary ecology of animal personality. Philos Trans R Soc B 365:4077-4088. https://doi.org/10.1098/rstb.2010.0182

Barber I, Hoare D, Krause J (2000) Effects of parasites on fish behaviour: a review and evolutionary perspective. Rev Fish Biol Fisher 10:131-165. https://doi.org/10.1023/A:1016658224470

Barber I, Huntingford FA (1995) The effect of Schistocephalus solidus (Cestoda: Pseudophyllidea) on the foraging and shoaling behaviour of three-spined sticklebacks, Gasterosteus aculeatus. Behaviour 132:1223-1240. https://doi.org/10.1163/156853995X00540

Barber I, Scharsack JP (2010) The three-spined stickleback-Schistocephalus solidus system: an experimental model for investigating host-parasite interactions in fish. Parasitology 137:411-424. https://doi.org/10.1017/S0031182009991466

Barber I, Svensson PA (2003) Effects of experimental Schistocephalus solidus infections on growth, morphology and sexual development of female three-spined sticklebacks, Gasterosteus aculeatus. Parasitology 126:359. https://doi.org/10.1017/S0031182002002925

Barber I, Walker P, Svensson PA (2004) Behavioural responses to simulated avian predation in female three-spined sticklebacks: the effect of experimental Schistocephalus solidus infections. Behaviour 141:1425-1440. https://doi.org/10.1163/1568539042948231

Bates D, Mächler M, Bolker B, Walker S (2015) Fitting linear mixedeffects models using lme4. J Stat Softw 67:1-48. https://doi.org/ 10.18637/jss.v067.i01

Bell MA, Foster SA (1994) The evolutionary biology of the threespined stickleback. Oxford University Press, Oxford

Beros S, Enders C, Menzel F, Foitzik S (2019) Parasitism and queen presence interactively shape worker behaviour and fertility in an ant host. Anim Behav 148:63-70. https://doi.org/10.1016/j.anbeh av.2018.12.004

Bode NW, Faria JJ, Franks DW, Krause J, Wood AJ (2010) How perceived threat increases synchronization in collectively moving animal groups. Proc R Soc Lond B 277:3065-3070. https://doi. org/10.1098/rspb.2010.0855

Boyer N, Réale D, Marmet J, Pisanu B, Chapuis JL (2010) Personality, space use and tick load in an introduced population of Siberian chipmunks Tamias sibiricus. J Anim Ecol 79:538-547. https://doi. org/10.1111/j.1365-2656.2010.01659.x

Demandt N, Praetz M, Kurvers RHJM, Krause J, Kurtz J, Scharsack JP (2020) Parasite infection disrupts escape behaviours in fish shoals. Proc R Soc B 287:20201158. https://doi.org/10.1098/rspb. 2020.1158

Demandt N, Saus B, Kurvers RHJM, Krause J, Kurtz J, Scharsack JP (2018) Parasite infected sticklebacks increase the risktaking behaviour of uninfected group members. Proc R Soc B 285:20201158. https://doi.org/10.1098/rspb.2020.1158
Dugatkin LA, FitzGerald GJ, Lavoie J (1994) Juvenile three-spined sticklebacks avoid parasitized conspecifics. Environ Biol Fish 39:215-218. https://doi.org/10.1007/BF00004940

Foster W, Treherne J (1981) Evidence for the dilution effect in the selfish herd from fish predation on a marine insect. Nature 293:466467. https://doi.org/10.1038/293466a0

Fox J, Weisberg S (2019) An R companion to applied regression, 3rd edn. Sage, Thousand Oaks, CA, https://socialsciences.mcmaster. $\mathrm{ca} / \mathrm{jfox} /$ Books/Companion/

Galton F (1907) One vote, one value. Nature 75:414. https://doi.org/ $10.1038 / 075414 \mathrm{a} 0$

Ghalambor CK, Reznick DN, Walker JA (2004) Constraints on adaptive evolution: the functional trade-off between reproduction and fast-start swimming performance in the Trinidadian guppy (Poecilia reticulata). Am Nat 164:38-50. https://doi.org/10.1086/ 421412

Giles N (1983) Behavioural effects of the parasite Schistocephalus solidus (Cestoda) on an intermediate host, the three-spined stickleback, Gasterosteus aculeatus L. Anim Behav 31:1192-1194. https://doi.org/10.1016/S0003-3472(83)80025-6

Giles N (1987) Predation risk and reduced foraging activity in fish: experiments with parasitized and non-parasitized three-spined sticklebacks, Gasterosteus aculeatus L. J Fish Biol 31:37-44. https://doi.org/10.1111/j.1095-8649.1987.tb05212.x

Godin J-GJ (1986) Antipredator function of shoaling in teleost fishes: a selective review. Nat Can 113:241-250

Gracia ES, de Bekker C, Hanks EM, Highes DP (2018) Within the fortress: a specialized parasite is not discriminated against in a social insect society. PLoS ONE 14:e0193536. https://doi.org/10. 1371/journal.pone.0193536

Gross J, Ligges U (2015) Nortest: tests for normality. R package version 1.0-4, https://CRAN.R-project.org/package=nortest

Ferrari N, Rosà R, Pugliese A, Hudson P (2007) The role of sex in parasite dynamics: Model simulations on transmissions of Heligmosomoides polygyrus in populations of yellow-necked mice, Apodemus flavicollis. Int J Parasitol 37:341-349. https://doi.org/ 10.1016/j.ijpara.2006.10.015

Hafer N, Benesh DP (2015) Does resource availability affect host manipulation?-An experimental test with Schistocephalus solidus. Parasitol Open 1:e3. https://doi.org/10.1017/pao.2015.3

Hafer N, Milinski M (2016) An experimental conflict of interest between parasites reveals the mechanism of host manipulation. Behav Ecol 27:617-627. https://doi.org/10.1093/beheco/arv200

Hammerschmidt K, Kurtz J (2009) Ecological immunology of a tapeworms interaction with its two consecutive hosts. In: Webster J (ed) Natural history of host-parasite. Academic Press, London, pp 111-137

Herskin J, Steffensen J (1998) Energy savings in sea bass swimming in a school: measurements of tail beat frequency and oxygen consumption at different swimming speeds. J Fish Biol 53:366-376. https://doi.org/10.1111/j.1095-8649.1998.tb00986.x

Holmes J, Zohar S (1990) Pathology and host behaviour. In: Barnard CJ, Behnke JM (eds) Parasitism and host behaviour. Taylor \& Francis Ltd, London, pp 193-229

Ioannou CC, Guttal V, Couzin ID (2012) Predatory fish select for coordinated collective motion in virtual prey. Science 337:1212-1215. https://doi.org/10.1126/science.1218919

Johansen J, Vaknin R, Steffensen JF, Domenici P (2010) Kinematics and energetic benefits of schooling in the labriform fish, striped surfperch Embiotoca lateralis. Mar Ecol Prog Ser 420:221-229. https://doi.org/10.3354/meps08885

Jolles JW, Mazué GP, Davidson J, Behrmann-Godel J, Couzin ID (2020) Schistocephalus parasite infection alters sticklebacks' movement ability and thereby shapes social interactions. Sci Rep 10:12282. https://doi.org/10.1038/s41598-020-69057-0 
Kalbe M, Eizaguirre C, Scharsack JP, Jakobsen PJ (2016) Reciprocal cross infection of sticklebacks with the diphyllobothriidean cestode Schistocephalus solidus reveals consistent population differences in parasite growth and host resistance. Parasit Vectors 9:1-12. https://doi.org/10.1186/s13071-016-1419-3

Koolhaas J, Korte S, de Boer S, van der Vegt B, van Reenen C, Hopster H, de Jong I, Ruis M, Blokhuis H (1999) Coping styles in animals: current status in behaviour and stress-physiology. Neurosci Biobehav R 23:925-935. https://doi.org/10.1016/S0149-7634(99) 00026-3

Krause J (1993a) Positioning behaviour in fish shoals: a cost-benefit analysis. J Fish Biol 43:309-314. https://doi.org/10.1111/j.10958649.1993.tb01194.x

Krause J (1993b) Transmission of fright reaction between different species of fish. Behaviour 127:37-48. https://doi.org/10.1163/ $156853993 X 00416$

Krause J, Godin J-GJ (1994) Influence of parasitism on the shoaling behaviour of banded killifish, Fundulus diaphanus. Can J Zool 72:1775-1779. https://doi.org/10.1139/z94-240

Krause J, Ruxton GD (2002) Living in groups. Oxford University Press, Oxford

Krebs JR, Davies NB (1993) An introduction to behavioural ecology, 3rd edn. Blackwell Scientific Publications, Oxford

Lafferty KD, Morris AK (1996) Altered behavior of parasitized killifish increases susceptibility to predation by bird final hosts. Ecology 77:1390-1397. https://doi.org/10.2307/2265536

Lafferty KD, Shaw JC (2013) Comparing mechanisms of host manipulation across host and parasite taxa. J Exp Biol 216:56-66. https:// doi.org/10.1242/jeb.073668

Lenth R (2020) Emmeans: estimated marginal means, aka least-squares means. R package version 1.4.8, https://CRAN.R-project.org/ package $=$ emmeans

Lester R (1971) The influence of Schistocephalus plerocercoids on the respiration of Gasterosteus and a possible resulting effect on the behavior of the fish. Can J Zool 49:361-366. https://doi.org/ 10.1139/z71-052

Lüscher A, Milinski M (2003) Simultaneous hermaphrodites reproducing in pairs self-fertilize some of their eggs: an experimental test of predictions of mixed-mating and hermaphrodite's dilemma theory. J Evol Biol 16:1030-1037. https://doi.org/10.1046/j.14209101.2003.00552.x

Marras S, Killen SS, Lindström J, McKenzie DJ, Steffensen JF, Domenici P (2015) Fish swimming in schools save energy regardless of their spatial position. Behav Ecol Sociobiol 69:219-226. https://doi.org/10.1007/s00265-014-1834-4

MATLAB (2017) Version 9.3.0.713579 (r2017b). The MathWorks Inc, Natick, MA

Milinski M (1990) Parasites and host decision-making. In: Barnard CJ, Behnke JM (eds) Parasitism and host behaviour. Taylor \& Francis Ltd, London, pp 95-116

Milinski M (1985) Risk of predation of parasitized sticklebacks (Gasterosteus aculeatus L.) under competition for food. Behaviour 93:203-216. https://doi.org/10.1163/156853986X00883

Muñoz JCV, Bierbach D, Knopf K (2019) Eye fluke (Tylodelphys clavata) infection impairs visual ability and hampers foraging success in European perch. Parasitol Res 118:2531-2541. https://doi.org/ 10.1007/s00436-019-06389-5

Noldus LP, Spink AJ, Tegelenbosch RA (2001) Ethovision: a versatile video tracking system for automation of behavioral experiments. Behav Res Methods Ins C 33:398-414. https://doi.org/10.3758/ BF03195394

Partridge BL (1982) The structure and function of fish schools. Sci Am 246:114-123. https://doi.org/10.1038/scientificamerican0682-114

Pascoe D, Mattey D (1977) Dietary stress in parasitized and nonparasitized Gasterosteus aculeatus L. Z Parasitenk 51:179-186. https://doi.org/10.1007/BF00500957
Pinheiro J, Bates D, DebRoy S, Sarkar D, R Core Team (2020) nlme: linear and nonlinear mixed effects models. $\mathrm{R}$ package version 3.1-148 , https://CRAN.R-project.org/package $=$ nlme

Pitcher TJ (1986) Functions of shoaling behaviour in teleosts. In: Pitcher TJ (ed) The behaviour of teleost fishes. Springer US, Boston, MA, pp 294-337. https://doi.org/10.1007/978-1-46848261-4_12

Pitcher TJ, Parrish T (1993) Functions of shoaling behaviour in teleosts, 2nd edn. In: Pitcher TJ (ed) Behaviour of teleost fishes. Croom Helm, London, pp 363-439

Poulin R (1999) Parasitism and shoal size in juvenile sticklebacks: conflicting selection pressures from different ectoparasites? Ethology 105:959-968. https://doi.org/10.1046/j.1439-0310.1999.00491.x

Poulin R (2000) Manipulation of host behaviour by parasites: a weakening paradigm? Proc R Soc Lond B 267:787-792. https://doi. org/10.1098/rspb.2000.1072

Prieto MH, López JCF, González RA, Salán ESM (2005) Geographical and temporal FA variation in threespine stickleback populations from Galacia (NW Spain). Evol Ecol 19:517-532. https://doi.org/ 10.1007/s10682-005-0883-6

Quinn T, Kendall N, Rich H, Chasco B (2012) Diel vertical movements, and effects of infection by the cestode Schistocephalus solidus on daytime proximity of three-spined sticklebacks Gasterosteus aculeatus to the surface of a large Alaskan lake. Oecologia 168:43-51. https://doi.org/10.1007/s00442-011-2071-4

R Core Team (2018) R: a language and environment for statistical computing. R Foundation for Statistical Computing, Vienna, Austria, https://www.R-project.org/

Richards EL, Van Oosterhout C, Cable J (2010) Sex-specific differences in shoaling affect parasite transmission in guppies. PLoS ONE 5:e13285. https://doi.org/10.1371/journal.pone.0013285

Ruxton GD, Jackson AL, Tosh CR (2007) Confusion of predators does not rely on specialist coordinated behavior. Behav Ecol 18:590 596. https://doi.org/10.1093/beheco/arm009

Schaerf TM, Dillingham PW, Ward AJ (2017) The effects of external cues on individual and collective behavior of shoaling fish. Sci Adv 3:e1603201. https://doi.org/10.1126/sciadv.1603201

Schärer L, Wedekind C (1999) Lifetime reproductive output in a hermaphrodite cestode when reproducing alone or in pairs: a time cost of pairing. Evol Ecol 13:381-394. https://doi.org/10.1023/A: 1006789110502

Scharf I, Modlmeier AP, Beros S, Foitzik S (2012) Ant societies buffer individual-level effects of parasite infections. Am Nat 180:671683. https://doi.org/10.1086/667894

Scharsack JP, Koch K, Hammerschmidt K (2007) Who is in control of the stickleback immune system: interactions between Schistocephalus solidus and its specific vertebrate host. Proc R Soc Lond B 274:3151-3159. https://doi.org/10.1098/rspb.2007.1148

Seppälä O, Karvonen A, Valtonen ET (2005) Impaired crypsis of fish infected with a trophically transmitted parasite. Anim Behav 70:895-900. https://doi.org/10.1016/j.anbehav.2005.01.021

Smyth J (1954) Studies on tapeworm physiology. vii. fertilization of Schistocephalus solidus in vitro. Exp Parasitol 3:64-71. https:// doi.org/10.1016/0014-4894(54)90019-3

Sumpter DJT, Pratt SC (2009) Quorum responses and consensus decision making. Philos Trans R Soc B 364:743-753. https://doi.org/ 10.1098/rstb.2008.0204

Tierney JF, Huntingford FA, Crompton DW (1993) The relationship between infectivity of Schistocephalus solidus (Cestoda) and anti-predator behaviour of its intermediate host, the three-spined stickleback, Gasterosteus aculeatus. Anim Behav 46:603-605. https://doi.org/10.1006/anbe.1993.1229

Tobler M, Schlupp I (2008) Influence of black spot disease on shoaling behaviour in female western mosquitofish, Gambusia affinis (Poeciliidae, Teleostei). Environ Biol Fish 81:29-34. https://doi. org/10.1007/s10641-006-9153-x 
Videler J (1993) Fish swimming. Chapman \& Hall, London

Ward AJW, Duff AJ, Krause J, Barber I (2005) Shoaling behaviour of sticklebacks infected with the microsporidian parasite, Glugea anomala. Environ Biol Fish 72:155-160. https://doi.org/10.1007/ s10641-004-9078-1

Ward AJW, Hoare DJ, Couzin ID, Broom M, Krause J (2002) The effects of parasitism and body length on positioning within wild fish shoals. J Anim Ecol 71:10-14. https://doi.org/10.1046/j.00218790.2001.00571.x

Ward AJW, Krause J, Sumpter DJT (2012) Quorum decision-making in foraging fish shoals. PLoS ONE 7:e32411. https://doi.org/10. 1371/journal.pone.0032411

Ward AJW, Sumpter DJT, Couzin ID, Hart PJB, Krause J (2008) Quorum decision-making facilitates information transfer in fish shoals. Proc Natl Acad Sci USA 105:6948-6953. https://doi.org/10.1073/ pnas.0710344105

Weber JN, Kalbe M, Shim KC, Erin NI, Steinel NC, Ma L, Bolnick DI (2017) Resist globally, infect locally? A transcontinental test of adaptation by stickleback and their tapeworm parasite. Am Nat 189:43-57. https://doi.org/10.1086/689597
Webster MM, Laland KN (2009) Evaluation of a non-invasive tagging system for laboratory studies using three-spined sticklebacks Gasterosteus aculeatus. J Fish Biol 75:1868-1873. https://doi.org/10. 1111/j.1095-8649.2009.02374.x

Wickham H (2016) Ggplot2: elegant graphics for data analysis. Springer-Verlag, New York. https://ggplot2.tidyverse.org

Wilson DS, Coleman K, Clark AB, Biederman L (1993) Shy-bold continuum in pumpkinseed sunfish (Lepomis gibbosus): an ecological study of a psychological trait. J Comp Psychol 107:250. https:// doi.org/10.1037/0735-7036.107.3.250

Wootton RJ (1976) The biology of the sticklebacks. Academic Press, London

Zuk M, McKean KA (1996) Sex differences in parasite infections: patterns and processes. In J Parasitol 26:1009-1024. https://doi. org/10.1016/S0020-7519(96)80001-4

Zuur A, Ieno EN, Walker N, Saveliev AA, Smith GM (2009) Mixed effects models and extensions in ecology with R. Springer, New York

Publisher's note Springer Nature remains neutral with regard to jurisdictional claims in published maps and institutional affiliations. 